\title{
Mask-interface for Continuous Positive Airway Pressure (CPAP) therapy: Selection and design considerations
}

\author{
Zhichao Ma ${ }^{\mathrm{b}}$, Michael Drinnan ${ }^{\mathrm{a}}$, Philip Hyde ${ }^{\mathrm{b}}$, Javier Munguia* \\ ${ }^{a}$ NHS Foundation Trust, Northern Medical Physical and Clinical Engineering \\ department, Newcastle upon Tyne, UK; ${ }^{b}$ Newcastle University, School of Engineering,
}

Newcastle upon Tyne, UK

*corresponding author: Javier Munguia (javier.munguia@newcastle.ac.uk)

\section{Introduction:}

Continuous Positive Airway Pressure (CPAP) therapy is a clinical treatment for moderate or severe Obstructive Sleep Apnea (OSA). Commercial CPAP mask designs normally come in standard sizes and the configuration is not commonly tailored to the patients' characteristics such as face topology, skin sensitivity and severity of OSA syndrome; however an optimal mask/interface selection is a key factor influencing the compliance and effectiveness of CPAP treatment.

\section{Areas covered:}

This review investigates conventional CPAP mask designs, their effect on OSA treatment and the related risk factors that can lead to skin damage after long term repeated use. It also introduces novel design-based approaches for customized CPAP masks which can overcome common issues associated with conventional masks. Through a literature search on common databases: SCOPUS, PubMed, Google Scholar, we identified reported facts on the influence of the mask interface, and current trends towards customized devices.

Expert Commentary: Recent literature on medical device-customization shows there is potential for optimizing the CPAP mask fit by adapting the interface to the patients' individual characteristics. This holds particularly true for users with abnormal features or simply outside conventional industry sizing standards. Enabling technologies for undertaking this adaptation include: Reverse engineering (3D Scanning), Computational Modelling and Additive Manufacturing. There is to date, no systematic method that integrates those elements into a standard solution, but a number of studies have shown their effectiveness for specific cohorts, including children hospitals and neonates units.

Keywords: CPAP therapy, mask selection, 3D printed CPAP masks, OSA syndrome

Statement of significance:

\section{INTRODUCTION}

\subsection{Sleep Disordered Breathing syndrome}

Sleep Disordered Breathing (SDB) is a common physical breathing condition characterized by intermittent hypoxemia and repeated arousals from normal sleep [1]. SDB can theoretically affect anyone, however it is most likely to occur in adults aged between 30 and 60 with a proportion of $4 \%$ of men and $2 \%$ of women [2] causing both short-term and long-term negative health consequences. Some immediate health issues 
are: sleep fragmentation, exaggerated fluctuations in blood pressure, intrathoracic pressure and heart rhythm [3]. Long-term effects include impairment in cognitive function, cardiovascular and hypertension morbidities, sudden death, and poor life quality [1, 2, 3, 4]. Additionally, increased risk of traffic accidents is also highly linked with the incidence of sleep-disordered breathing syndrome [5].

\subsection{Obstructive Ssleep Apnea}

There are a number of conditions that can fall into a SDB umbrella classification such as: Apnea, Hypopnea, Obstructive Sleep Apnea (OSA), Central Sleep Apnea (CSA), Sleep related hypoxemia and primary snoring, among others [6]. However SDB can be mainly classified into two categories: CSA and OSA [7] with the latter being the most common type [8] . OSA is defined as a 'sleep-related breathing disorder that involves a decrease or complete halt in airflow despite an ongoing effort to breathe' [9], mainly due to airways collapsing due to muscle relaxation during sleep. A common measurement of sleep apnea is the Apnea-Hypopnea Index ( $\mathrm{AHI}$ ), which is the number of episodes of Apnea or Hypopnea occurred per hour during sleep [2]. From a clinical perspective, Apnea is the brief periods of breathing cessation and Hypopnea is the marked reduction in tidal volume [2]. The collapse of upper airway during sleep is estimated as the main reason leading to OSA syndrome [10]. In normal breathing conditions, the upper airways are open during sleep allowing fresh air to flow from nose to lung. However, for OSA these same airways are physically blocked due to the upper soft tissue falling back to the throat consequently stopping the normal airflows. Figures 1 and 2 show the normal breathing and obstructed breathing schematic respectively [11].

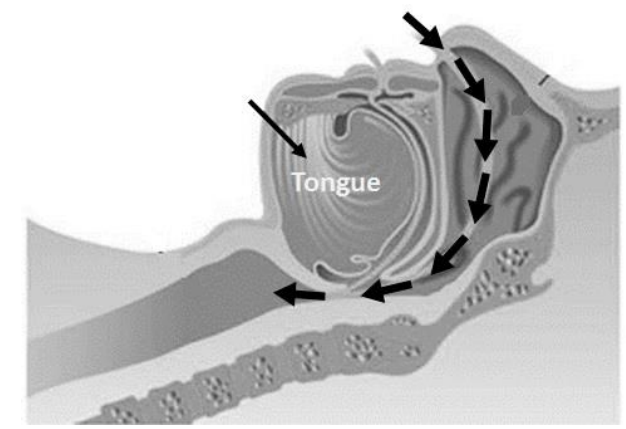

Figure 1. Airways opened during normal breathing

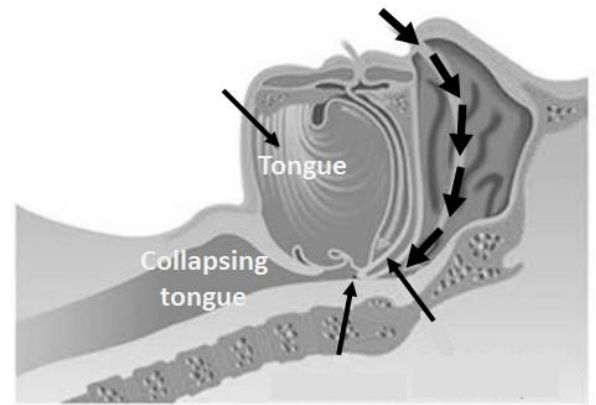

Figure 2. Obstructed breathing as a result of soft tissue collapsing

Mild OSA is typically defined by an AHI number between 5 and 15 , whereas moderate OSA syndrome ranges between 15 and 30 . Severe OSA syndrome on the other hand is classified by an AHI number over 30 [12]. Some potential risk factors for OSA include: obesity, ageing and increase in neck circumference [7]. Common treatment options besides the provision of a PAP device include: avoiding alcohol, weight loss, use of dental appliances, surgery and pharmacological management [7]. Amongst these 
treatments, Positive Airway Pressure (PAP) has been the most widely used to treat moderate or severe OSA syndrome for decades.

\subsection{Continuous Positive Aairway Pressure therapy}

Positive Airway Pressure therapy has been used to treat OSA syndrome as an effective clinical approach. There are a wide range of PAP devices such as: Continuous Positive Airway Pressure (CPAP), Auto adjusting CPAP, Bi-level PAP, adaptive servo ventilation and volume-assured pressure support [13]. BiPAP and CPAP are two typical forms, which are commonly used for treating moderate or severe OSA syndrome and both rely on supplying pressurized air to the patient's airways via a mask. BiPAP can offer different levels of air pressure during inspiration and expiration; typically, higher air pressure is supplied during inspiration and lower pressure is supplied during expiration as opposed to CPAP which delivers a constant single pressure level. The CPAP system comprises a pump, a flexible hose, a facial mask and a headgear. Figure 3 shows a basic connection between CPAP device and patients $[14,15]$. The pump is used to pressurize the air beyond atmospheric pressure, at a level that is comfortably tolerated by the wearer [16] opening the blocked airway via a pressurized airflow (Figure 4).

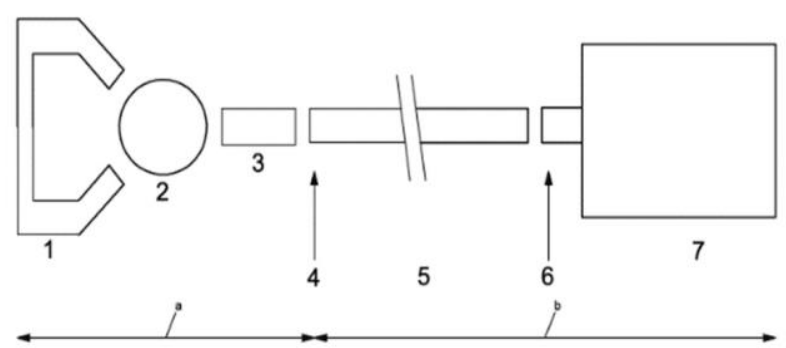

Figure 3 CPAP system schematic: 1) Headgear, 2) CPAP mask, 3) Connection, 4) Patient connection port, 5) Tubing, 6)Gas output port connector, 7)CPAP equipment (with or without humidifier) The exhaust port can be located in the connecting element (3) or the mask (2)

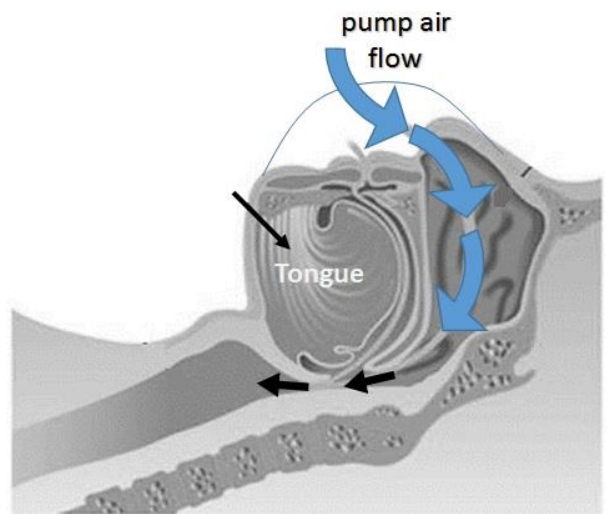

Figure 4 Working principle of CPAP therapy with pressurized air unblocking the collapsed soft tissue

CPAP therapy has been commonly used to treat moderate or severe OSA syndrome since it was initially invented by Colin Sullivan in 1980 in Australia [17] by delivering constant positive air pressure through patient's nose and throat, thus opening the blocked airway. Auto adjusting CPAP devices can automatically adjust the air pressure level based on respiratory events, but keep the same pressure throughout the respiratory cycle. Auto adjusting CPAP benefits patients who may need a higher-pressure support due to sudden rapid eye movement (REM) or supine position during sleep, but cannot tolerate this higher-level air pressure throughout the entire night [13]. 


\subsection{CPAP beyond Sleeep Apnea}

CPAP has also been widely considered as a form of Non-Invasive Ventilation particularly in cases of acute respiratory failure such as: patients with chest wall trauma, acute pneumonia, hypoxaemia resistant to high flow oxygen [18]. The use of CPAP devices has also been reported in the context of acute cardiogenic pulmonary edema, immunodeficiency-related pneumonia and cases of abdominal surgery [19].

\section{COMMON CPAP MASK CONFIGURATIONS}

\subsection{Commonly prescribed CPAP masks}

A variety of CPAP masks-interfaces are commercially available in standard configurations and can be generally classified into five categories depending on the way the pressurized air is supplied: Oronasal (Full-faced) masks, Nasal masks, Nasal pillows, Oral masks, and Total face masks. CPAP masks are selected based on: past clinical effectiveness, patient's individual characteristics, previous experience of medical devices and economic aspects [20]. However the ultimate criteria for mask selection is the patient's feel and preference, particularly after having tried a specific model for periods ranging between 3 and 6 months [21]. Table I shows a comparison amongst different types of facial masks in term of contact area and air delivery path [15, 22, 23]. The main components that conform a standard CPAP mask include: frame, cushion, headgear, forehead pad, tubing, elbow assembly and nasal pillows. The configuration of each component depends on the different CPAP device manufacturers, however, as most of the CPAP mask elements are mass produced, the designs, materials and sizes tend to be standardized.

Table 1. Characteristics of different types of CPAP interfaces [13, 16-17]

\begin{tabular}{|c|c|c|c|}
\hline $\begin{array}{c}\text { Mask/ } \\
\text { Characteristics }\end{array}$ & Contact area & Air delivery path & Sample image \\
\hline $\begin{array}{l}\text { Oronasal (full- } \\
\text { faced) masks }\end{array}$ & $\begin{array}{l}\text { Covers both nose and } \\
\text { mouth }\end{array}$ & $\begin{array}{l}\text { Air flows through nose and } \\
\text { mouth }\end{array}$ & \\
\hline Nasal masks & Covers nose only & Air flows through nose only & \\
\hline Nasal pillows & $\begin{array}{l}\text { Contact inside rim of the } \\
\text { nostrils }\end{array}$ & Air flows through nose only & \\
\hline Oral masks & Covers mouth only & $\begin{array}{l}\text { Air flows through nose and } \\
\text { mouth }\end{array}$ & \\
\hline
\end{tabular}




\begin{tabular}{|l|l|l|l|}
\hline Total face masks & Covers entire face & $\begin{array}{l}\text { Air flows through nose and } \\
\text { mouth }\end{array}$
\end{tabular}

\subsection{Mask selection and impact on CPAP therapy}

Mask selection is a crucial factor challenging the success of CPAP therapy. The choice of CPAP mask is decided by multiple factors, such as patient's individual characteristics (facial features, age, gender, sleeping habits and health condition), different breathing pattern (mouth and/or nose breather), the severity of OSA syndrome (mild / moderate /severe level), and the frequency and length of use of CPAP interface [20]. It has been reported that nearly half of OSA patients either suspend or permanently quit the CPAP therapy due to interface related side effects [24].

\subsubsection{Oronasal (Full-faced) masks.}

Main features and utility

Oronasal masks mainly cover the patient's nose and mouth during the treatment. Oronasal (Full-faced) masks have been suggested as the first choice for non-invasive ventilation [25]. One of the main advantages with the use of Oronasal masks is the prevention of unintentional mouth air leak. These masks are normally prescribed for mouth-breathers and OSA patients who experience air-leaks with nasal delivery masks [26, 27].

Reported issues and side-effects

Some reported negative effects of Oronasal masks include facial skin damage/discomfort caused by the longstanding use of the full-face interface [20], as well as localized skin damage, such as red marks and pressure sores, mainly caused by repeated tissue loading from the CPAP mask interface $[20,28,29]$. Over-tightening straps is a common action by CPAP users to compensate the unintentional air leakage during treatment, thus increasing the contact pressure around the mask-facial contour [28, 29]. Facial skin problems can consequently be developed with the use of Oronasal masks during treatment. Due to the configuration of the Oronasal mask, the most sensitive areas where facial skin problems may be developed include the nasal bridge and paranasal regions, right and left cheeks, and the lower part of the mouth area [30].

\subsubsection{Nasal masks and Nasal pillows}

Main features and utility

The use of nasal masks in CPAP treatment has increased significantly with a range of benefits being reported by recent studies; Ebben et al. [31] suggested that the use of nasal masks leads to a better treatment tolerance and less residual AHI compared to the use of Oronasal masks. In addition, the need for higher-pressure support is normally required in Oronasal masks compared to nasal masks [26, 32]. Some studies have proposed that nasal masks can be alternatives for Oronasal masks in CPAP treatment, 
as there is less opportunity of developing facial side effects [26, 32]. The performance between nasal masks and nasal pillows has also been discussed in terms of the adherence, comfort, air leakage and facial side effects. Clifford et al. [33] suggested that nasal pillows provide a better tolerance and fewer side effects compared to the use the nasal masks. Zhu et al [34] found that nasal pillows have the same effectiveness as nasal masks even at a high CPAP pressure. Enhanced comfort and lower facial-pressure due to the use of nasal pillows have been reported by De Araujo et al. [35].

Reported issues and side-effects

Although the use of nasal masks can reduce the contact area between mask interface and patient's facial skin, other facial side effects can also be developed with the use of nasal masks in CPAP treatment such as nasal dryness [36] and congestion developed with the prolonged use of the nasal interface [35]. However, facial side effects with the use of nasal pillows, such as nasal dryness, bleeding, and headaches, negatively impact on its performance in CPAP therapy [35, 37]. In addition, pressure sore inside the nose caused by the insertion of unmatched nasal pillows has also been reported, thus affecting the performance of nasal pillow in CPAP treatment, [38].

\subsubsection{Oral masks}

Main features and utility

Oral masks, which only cover a patient's mouth, have been used in treating OSA syndrome for decades. The use of Oral masks in CPAP treatment is less common compared to Oronasal and nasal masks and there are few studies comparing the effectiveness and compliance between these types of interfaces.

Reported issues and side-effects

No significant differences have been established in terms of effectiveness, pressure levels, comfort and compliance to CPAP treatment between Oral masks and Oronasal or nasal masks. However, side effects resulted by using Oral masks have limited its popularity particularly those associated with dry mouth, dental pain and nasal air leak $[39,40,41,42]$.

\subsubsection{Total face masks}

Main features and utility

The use of total face masks as intervention in CPAP treatment has been suggested as effective alternative to the use of Oronasal masks and nasal masks [43]. Total face masks are designed to cover patient's entire face, hence avoiding direct contact with sensitive facial areas where skin damage is easily developed, such as the nasal bridge, upper lip, and cheeks. Total face masks use has been reported in the context of acute respiratory failure or as a result of emergency-hospital interventions [44]

Reported issues and side-effects

Masks which tend to cover the totality of the face are not commonly prescribed by clinicians unless they respond to a major health risk associated with a chronic breathing condition, and these tend to be less common commercially available options [45].

\section{Determinants for development of skin related effects from CPAP treatment}

Although CPAP therapy has been widely used and has been proven an effective clinical approach to treat moderate or severe OSA syndrome, interface-related facial effects, such as discomfort, unintentional air leaks and facial skin problems, and tend to reduce the overall effectiveness of CPAP therapy. Excessive pressure exerted from the CPAP mask to patient's face is considered as the main risk factor leading to some of the most notable skin-related side effects. There are several other factors closely linked with the 
formation of skin damage at the interface between patient's facial skin and CPAP mask, including: non-suitable CPAP mask configuration and poor fitting, allergic reaction to materials used in the CPAP mask and development of a moist microclimate between skin and mask interface. Additional factors include consideration of patient's individual characteristics such as facial features, allergies history, severity of OSA syndrome, as well as experience on the use of CPAP devices.

\subsection{CPAP interface related facial side effects}

CPAP interface-related facial side effects, such as discomfort, unintentional air leakage, and facial skin problems have consistently affected the overall effectiveness of CPAP treatment for patients with OSA syndrome [20, 24, 26]. In fact, CPAP interface-related facial side effects, such as local skin pressure sores around the mask or inside the nose, dry mouth and/or nose, headache, nose bleeding, allergic reactions and dental pain can be developed with the use of different type of CPAP masks [28, 30, 35, 33, 38, 43]. Table II summarizes some typical CPAP interface related facial side effects with the use of different CPAP masks.

Table 2. Typical interface-related facial side-effects [15, 22, 24, 27, 28, 31, 36]

\section{Mask types}

\begin{tabular}{|l|l|}
\hline Oronasal (Full-faced) masks & $\begin{array}{l}\text { Local skin damage (mainly red marks and pressure } \\
\text { sores) around the mask (particularly at the nasal } \\
\text { bridge and lateral side of nose) }\end{array}$ \\
\hline Nasal masks & $\begin{array}{l}\text { Dry nose/mouth/ throat, local skin damage around } \\
\text { the mask (mainly on the lateral side of nose) }\end{array}$ \\
\hline Nasal pillows & $\begin{array}{l}\text { Pressure sore inside the nose, nasal dryness, nose } \\
\text { bleeding, nose congestion and headache }\end{array}$ \\
\hline Oral masks & $\begin{array}{l}\text { Dry mouth, dental pain, local skin damage around } \\
\text { the mask }\end{array}$ \\
\hline Total face masks & \begin{tabular}{l} 
Local skin damage around the mask \\
\hline
\end{tabular} \\
\hline
\end{tabular}

\subsection{CPAP mask configuration and fitting}

One of the main drawbacks of the CPAP-mask related intervention is the lack of sizing and fitting options which conform to the wearer's face. Some sizing templates provided by CPAP device manufacturers only measure several 2D facial features such as the distance between eyes, mouth width, nose length as well as the distance between cheekbones, for selecting a right fitting CPAP mask interface. However, such plain fitting criteria can neglect the 3 dimensional nature of human face with relevant features such as the slope and angle of nose, bony prominences, inherit asymmetries and tissue displacement at different postures, gestures or activities. Some '3D facial features' present significant differences across individuals which are not noticed by standard 2D sizing templates, which may increase the probability of adopting an ill-fitting mask, leading to potential sources of air leakage through the gap between mask and face. Due to the poor fit, the headgear strap is also often overly tightened as a means to compensate and correct the fit, thus increasing the discomfort level and overall contact pressure [20].

\subsection{Common Materials for CPAP mask}

Although not commonly acknowledged in literature or previous studies, allergic skin reactions to the soft materials at the mask interface are a recurring issue in daily practice 
[21]. A variety of materials can be used to form a CPAP mask with the most commonly used being Silicone-based polymers, such as Silicone elastomer and Silicone rubber. These are normally over-molded to a hard-shell plastic 'dome' to provide a conformable and soft skin interface. However, most used Silicone-based materials are impermeable and non-breathable, which can lead to an increase in humidity between face and mask [29]. Increased humidity has been linked to the development of facial pressure ulcers as the repeated mechanical loading between mask and face has a major effect on moist tissue [46]. Gel-based CPAP masks have been suggested as alternatives to Silicone interfaces as these can automatically mold themselves to the patient's facial contour, thus potentially securing a tight fit. However, the use of gel masks also requires a tight adjustment of straps as the gel structure will tend to re-form and re-balance as tissue is displaced by natural face movement. A third option is foam, cloth and other CPAP mask designs including fabrics/textiles to seek a better fit and comfort. However material selection has proven to be a qualitative process refined and informed by specific patient testimonials and usage history.

\subsection{Microclimate-induced discomfort}

In CPAP therapy, the microclimate change between patient's facial skin and CPAP mask is an important consideration for manufacturers, users and patients' groups, although not commonly studied in related CPAP literature [47, 48]. Humidification at the interface is not only dependent on the mask material but it is inherently related to the wearer's environment, external climate, sleeping habits and preferences $[49,50]$. Moreover, age seems to be an added factor to the microclimate-based discomfort, with patients over 60 normally requiring heated humidification [51]. Excessive pressure, shear, and friction applied to patient's facial skin can cause facial pressure ulcers and shorten the CPAP therapy length and tolerance [46]. Excessive temperature and moisture can also increase the risk of the occurrence of facial pressure ulcers; It has been reported that the risk of facial tissue damage can be highly increased when local skin temperature over $35^{\circ} \mathrm{C}$ [29]. Condensation inside the CPAP mask and inside the breathing tube are also typical factors creating a non-ideal microclimate environment.

\subsection{Variations on individual characteristics and skin properties.}

Human skin properties, such as elasticity, moisture and thickness of facial tissue, are diverse among users/patient. Aged groups are more prone to present skin damage during CPAP treatment as skin elasticity, thickness and resistance are mainly affected by ageing [52]. Infants have also been identified with high risk for nasal damage in CPAP treatment [38]. The occurrence of facial side effects can be increased with a longer time of use with CPAP masks depending on the severity of the OSA syndrome. In addition, a poor education, understanding or ill-informed prescription for the use of CPAP devices also affect the effectiveness of CPAP treatment.

\section{DESIGN AND MANUFACTURE TRENDS}

A number of strategies have been adopted in order to minimize any adverse effects that could be potentially caused by the CPAP mask interface. The UK's National Institute for Health and Care Excellence (NICE) [53] established since 2008 a widespread adoption of CPAP as an effective technology treatment for Obstructive Sleep Apnea /Hypopnea Syndrome (OSAHS) [54]. This motivated various agencies such as the Association for Respiratory Technology and Physiology (ARTP) to produce specific guidelines [54, 55] for the provision of effective masks. The criteria and specific design and manufacturing aspects suggested by ARTP are shown on Table III. Although these guidelines recognize that custom-made masks are not a common nor readily available across many healthcare service, the acknowledgement of custom masks across different public and official regulatory bodies, opens the doors to new developments in the field.

Table 3. Mask design requirements and considerations [39] 


\begin{tabular}{|c|c|}
\hline Mask/interface aspect & ARTP requirement \\
\hline Materials & $\begin{array}{c}\text { *Mask/interface must be as soft and pliable as possible to provide } \\
\text { maximum patient comfort. } \\
\text { *Materials independently certified as being medical grade, } \\
\text { hypoallergenic substances }\end{array}$ \\
\hline Size(s) & ${ }^{*}$ must be available in a range of sizes \\
\hline Adjustment & $\begin{array}{c}\text { All masks must be fully adjustable including considerations for } \\
\text { adjustable for height, reach and rake }\end{array}$ \\
\hline $\begin{array}{l}\text { Disassembly, Cleansing } \\
\quad \text { and Reassembly }\end{array}$ & $\begin{array}{l}\text { Must be capable of being disassembled and conversely capable of } \\
\text { being correctly re-assembled by the same user for the purposes of } \\
\text { cleansing all component parts. }\end{array}$ \\
\hline $\begin{array}{l}\text { Re-use/Single Patient } \\
\text { Use }\end{array}$ & $\begin{array}{c}\text { required by legislation to be marked as either reusable or single patient } \\
\text { use } \\
\text { Reusable masks must be able to withstand disinfection/autoclaving to } \\
\text { recognized hospital standards }\end{array}$ \\
\hline $\begin{array}{c}\text { Connection of CPAP } \\
\text { Machines }\end{array}$ & $\begin{array}{l}\text { It must be possible to connect a CPAP machine from any } \\
\text { manufacturer/vendor to any CPAP mask/interface without adaptors }\end{array}$ \\
\hline $\begin{array}{l}\text { Lock/Release } \\
\text { Mechanisms }\end{array}$ & $\begin{array}{c}\text { All full face masks should have a quick-release mechanism and must } \\
\text { comply with safety standards that avoid risk of asphyxiation if the } \\
\text { device fails or there is a power-cut }\end{array}$ \\
\hline $\begin{array}{l}\text { Assembly at Point of } \\
\text { Sale }\end{array}$ & Masks/Interfaces should, where possible, be supplied fully assembled \\
\hline Individually Made Masks & All previous points apply \\
\hline
\end{tabular}

\subsection{NEW DEVELOPMENTS FOR CPAP MASKS: CUSTOMIZATION}

Although not commercially available, a number of customized CPAP mask deigns have been reported in literature as a strategy to treat specific and complicated cases such as facial deformities or asymmetries [56] or special purpose CPAP masks for specific cohorts [57] and documented clinical cases requiring special adaptations such as children masks [58, 59]. For conventional mass produced CPAP interfaces, mask configuration and sizing are dictated by standard proprietary sizing measures (extrasmall size, small size, middle size, large size and extra-large size), however OSA patients with different physical characteristics can only select masks from pre-defined templates, which increases the risk of wearing over-tensioned masks for prolonged period's thus increasing discomfort, promoting air leakages and potentially leading to skin problems.

Recent attempts to adopt novel digital technologies for the development of custom-made CPAP masks have been reported in literature but are still scarce. The Children's Hospital Medical Centre (Cincinnati, US) is the first the report a series of user trials of custom 3D Printed CPAP masks for pediatric patients in a 6-month pilot study that uses digital techniques such as 3D scanning (Artec, USA) and 3D printing for the fabrication of madeto-measure devices [60]. Cheng et al [61] have used a rapid tooling (RT) technique to manufacture a customized nasal CPAP mask cushion. Customized nasal CPAP mask cushion design has been done based on the 3D facial model. Objet's Polyjet and shape deposition manufacturing (SDM) have been used to fabricate the cushion mode respectively. Finally, the customized nasal mask cushion was obtained by silicone casting.

Morrison et al [56] discussed the fabrication of a customized CPAP mask cushion using 3D scanning and additive manufacturing. The specific facial data was obtained using 3D photography and the customized full-faced mask cushion, which covered the patient's mouth and nose, was designed based 3D CAD software (Mimics, Materialise, Belgium). A custom negative-mask was 3D printed (Objet Connex) and silicone was casted into 
the mold obtaining a customized mask insert. Results over a sustained 3-month period demonstrated a noticeable improvement in comfort, air-leakage reduction, and a $24 \%$ decrease in residual Apnea-Hypopnea Index (AHI).

Although previous attempts have proved the feasibility of fabricating a customized CPAP mask based on reverse engineering, computational modelling and AM technology, there is limited evidence of the resulting performance of the custom devices. Furthermore several challenges remain such as available materials selection, influence of microclimate between mask interface and facial skin, as well as users' reports about skin interaction and improvements over longer periods. In the drive towards customization of medical devices there is also a 'design' element which remains unsolved, as every custom device requires a tailored strategy from measuring to custom manufacturing and fitting. Munguia \& Ma [62] laid out a computational design mapping process for the design, fit simulation and manufacture of custom CPAP masks, with one of the main challenges being the correct definition of facial-contour features extracted from a 3 dimensional mesh. Two of the main future drivers for customization are described next: Computational modelling and Additive Manufacturing (3D printing).

\subsubsection{Computational Modelling}

There have been various attempts on the use of computer modelling and simulation for custom respiratory devices [63, 64, 65] Barros et al [30] conducted an experiment to study the facial skin damage zones caused by excessive pressure resulting from Oronasal masks. Through computational simulation, they indicated that the most sensitive facial pressure zones, where pressure ulcers were easily developed, were the nasal bridge and paranasal regions respectively. They also pointed out that the variation in facial soft tissue caused a direct impact on the total amount of pressure applied.

One of the main limitations of this approach is the availability of full 3D CT models which include multilateral information for skin, bone, fat, soft tissue, etc. to provide a realistic responses to the desired loading regime. The approach adopted by Morrison et.al [56] relied on face 3D scanning, which provides a fully detail 3 dimensional mesh of the patient's face but lack any materials data and depth. Merging both of these technologies: 3D scanning and computational Finite Element Analysis, can have the effect of providing realistic simulations for fit and comfort on an individual basis.

\subsubsection{Additive Manufacturing}

Additive manufacturing (AM) has been increasingly used on medical device fabrication for the past 10 years. Also widely recognized as Rapid prototyping, 3D printing or Solid Freeform Fabrication [66], AM technology has been suggested as a promising technology that can revolutionize the custom medical device field $[67,68,69]$. This novel manufacturing technology differs from conventional manufacturing processes, such as subtractive processes (milling or drilling), joining processes (casting or forging) and formative processes (welding or fastening) in the way material is deposited at specific locations on-demand [70, 71].

Manufacturing via AM has been reportedly instrumental in the launch of two recent ventures devoted to the design and fabrication of custom-made 3D-printed CPAP masks: Metamason (USA) [72] and Inspirate Medical (Singapore) [73], with both parties claiming on progress towards FDA approval of their respective products. Although other 3D printed devices have previously received FDA $510(\mathrm{k})$ clearance $[74,75]$ there are no FDA -cleared available 3D-printed CPAP devices. Recent research in Newcastle University [76] demonstrated the potential for manufacturing medical devices using a redistributed supply chain, powered by digital design and manufacturing. Future and ongoing work is based on the potential for designing, simulating and manufacturing CPAP devices by the development of a method in which a 3D digital representation of individual's face is matched to an optimum fitting CPAP interface while at the same time 
being able to predict peak pressure and potential leakage levels before the manufacture of the final device.

\section{Expert commentary}

The use and prescription of CPAP mask therapy remains the 'gold' standard for the treatment of OSA. Regardless of the CPAP mask configuration, size, material or brand there are common pitfalls in the fitting and long-term use of the device which hamper effectiveness of the CPAP treatment. Basically an ill-fitting device will lead to a quick rejection of the treatment, leading to a succession of follow-up visits to the clinician in charge, thus potentially leading to an increase in the overall cost for the provision of CPAP devices. There are clear differences in the provision of CPAP pumps and interfaces across different healthcare systems, e.g. in the USA CPAP therapy can be covered by private insurance while in countries like UK or Spain it is provided by national healthcare systems. However regardless of the scenario, a good mask/interface fit can provide patients a quicker recovery after being prescribed the therapy.

Table 1 documented some of the most common mask interfaces currently being prescribed and it will depend on the individual patient's preference, OSA severity and the clinician's diagnose to choose the most convenient type. However, regardless of the final selection, the mask-interface fit and perceived comfort will ultimately dictate whether the treatment will be followed or interrupted. The ways CPAP masks are provided and evaluated are dictated by the different healthcare systems. Whereas in places like the US and Australia CPAP therapy can be covered by private insurance, in the UK most masks are provided via the National Health Service (NHS), therefore the final mask selection will also be subjected to supply, coverage, availability and inventories of each different NHS trust.

Mask-interface selection has played an important role determining the success of CPAP treatment and it is not uncommon for patients to try multiple design, sizes and configurations before sticking to the best performing device. The limitations showed by conventional CPAP masks, such as averaged configuration, limited size ranges and nonideal material selection, considerably impact their performance during treatment. Facial side effects associated by wearing an ill-fitted CPAP mask can be minimized by using a customized CPAP mask, however available studies are scarce and tend to be focus on case studies such as pediatric patients in need of sizes or patients with facial abnormalities which deem standard mask designs unusable. The pathways towards custom made medical device provision seems to be gradually opening to new fields such as prosthetics and orthotics, however this is true only in cases where the custom device can be cost-effectively manufactured in-house, which is not a common feature for most medical systems and it requires the elaboration of a business case to justify the viability of the intervention.

Reverse Engineering, Computational Modelling, and Additive Manufacturing can offer a bespoke and cost effective solution for the development of customized CPAP masks, and the use of such tools within healthcare environments is becoming a reality gradually. This coupled with the capabilities of virtual CAD/FEA analysis can lead to a new breed of devices which are virtually pre-tested before manufacture, guaranteeing and appropriate fit.

\section{Five-year view}

While there is a wide variety of state of the art literature of CPAP efficiency studies, measuring the air pressure performance, valve comparisons, severity-based prescription criteria, the design of the interface has received limited attention. A number of studies 
from other fields such as performance sports garments, aviation devices and emergency equipment can be brought into the CPAP mask design, however there is one element which adds to the overall complexity of this device, which is the long-term use, sometimes the permanent use during the patient's lifetime. This means that the development of such devices must also include research elements from qualitative comfort research, user focus groups outcomes, as well as design theory and materials science, so that the user's input is considered for more effective designs.

A number of Hospitals across the UK and worldwide are increasingly embracing digital design, modelling and manufacturing and their capabilities are extending to the manufacture and provision of devices such as writ splints, bionic arms, home-use, uroflow meters, and foot/ankle orthotics. Some of the pilot studies included in this review hold the promise of the possibility to design and manufacture custom-made devices particularly for children, and more specifically for neo-nates, whose skin sensibility has been acknowledged as being particularly affected by ill-design and ill fitted conventional breathing devices. We believe the success in the design and development of custom CPAP mask interfaces will rely on the evidence acquired after prolonged recorded use, but this is more like to come from specialized hospital units, Universities and research institutes rather than from commercial ventures; this implies that the pathways to the development of such custom devices will be in direct proportion to the speed of adoption of digital design, development and manufacturing technologies necessary, plus building the necessary expertise within the institutions.

\section{KEY ISSUES:}

- This is one of the first reviews purely focused on the face-mask interface and the relevance of the design features linked to patient preferences.

- No CPAP mask design is exempt of potentially causing skin damage, particularly when the tissue loading regime is part of a long term treatment.

- High tightening pressure is normally one of the top causes of patient discomfort, and it is linked to an ill-fitting device which has been over tightened to compensate the misalignment with the face.

- Leaks is another resulting effect from ill-fitting masks, which directly affects the overall therapy as the pump pressure level defined during consultation may be reduced as a result or pressurized air not being effectively supplied.

- Although mask interface materials and skin compatibility is not a normally discussed factor, it has been found to be a recurring issue in clinical practice, which makes clinicians consider alternative designs/providers according to each case.

- Medical device-customization has been a recent trend across different medical fields such as orthotics, prosthetics and prescription devices, however recent attempts for customizing mask interface have not yet arrived to a wider market.

- We foresee the use of computational modelling linked to imaging technologies such as 3D scanning can help clinicians inform their practice by establishing "best-fit" criteria prior to selecting an appropriate breathing device. 
[1] Yaffe K, et al. "Sleep-disordered breathing, hypoxia, and risk of mild cognitive impairment and dementia in older women," Journal of the American Medical Association 306:613-9, 2011

[2] Flemons W. "Obstructive sleep apnea.," New England Journal of Medicine, vol. 347: 498-504, 2002

[3] Somers V, et al. "Sleep apnea and cardiovascular disease," Journal of the American College of Cardiology, 52:686, 2008

[4] Peppard P, et al. "Increased prevalence of sleep-disordered breathing in adults" American journal of epidemiology, 177:1006-1014, 2013

[5] Teran-Santos J, et al. "The association between sleep apnea and the risk of traffic accidents," New England Journal of Medicine.340:847-851, 1999

[6] Patil S. "Current Definitions for Sleep," FDA workshop on medical devices for SDB, 2018

* Clarifies medical-device related terminology relevant to sleep/breathing disorders

[7] Ho M, and Brass M. "Obstructive sleep apnea". Neurology international, vol. 3, 2011

[8] Morgenthaler T, et.al. "Complex sleep apnea syndrome: is it a unique clinical syndrome?" Sleep, vol. 29: 1203-09, 2006

[9] AASM, "American Academy of Sleep Medicine. Obstructive Sleep Apnea," 2008. [Online]. Available: https://aasm.org/ [Accessed 3108 2018]

[10] Kuna S, and Sant'Ambrogio G. "Pathophysiology of upper airway closure during sleep" Jama, 266:1384-89, 1991

[11] Doyle C. "The Engineering of Masks for Application in Continuous Positive Airway Pressure (CPAP) Therapy," Newcastle University MSc Archive, 2017

[12] Lloberes $P$, et al. "Diagnosis and treatment of sleep apnea-hypopnea syndrome" Archivos de Bronconeumología, 2011

[13] Johnson K. "Treatment of sleep-disordered breathing with positive airway pressure devices: technology update". Medical Devices (Auckland, NZ), 8, p.425, 2015

** Review of CPAP treatment and their implications to breathing disorders

[14] ISO17050-1:2004, "Conformity assessment - Supplier's declaration of conformity - Part 1: General Requirements.," International Standards Organization, 2004

[15] Fisher and Paykel, Catalogue, Available from https://www.fphcare.co.nz

[16] ML H and SD B. "Obstructive sleep apnea," Neurol Int., vol. 3,15, 2011

[17] Sullivan C and Issa F. "Pathophysiological Mechanisms Obstructive Sleep Apnea," Sleep, Vols.3:235-46, 1980 
[18] British Thoracic Society, "BTS/ICS Guidelines for the Ventilatory Management of Acute Hypercapnic Respiratory Failure in Adults," British Thoracic Society, 2017. Available: https://www.brit-thoracic.org.uk/standards-of-care/guidelines/

* Explores the acute interventions where CPAP mask interfaces are also widely used

[19] Mistraletti $G$ et al. "Noninvasive CPAP with Face Mask: Comparison" RESPIRATORY CARE, vol. 58, no. 2, 2013

[20] Brill A. "How to avoid interface problems in acute noninvasive ventilation" Breathe, Vol 10, 230-242, 2014

[21] Freeman Hospital, Lung Function Dept. Newcastle upon Tyne Hospitals NHS Foundation Trust 2018. [Interview], 2018

[22] Resmed. Respiratory Product series, 112017 Available: https://www.resmed.com/uk/en/consumer/products.html

[23] Philips. Philips Sleep Apnea Masks, 2017. Available: http://www.philips.co.uk

[24] McLornan P, et al. "Customizing a nasal CPAP mask using a silicone elastomer" The Journal of prosthetic dentistry, vol. 100, 147-152, 2008

[25] Crimi C, et al. "A European survey of noninvasive ventilation practices" European respiratory journal, vol. 36, 362-69, 2010

[26] Borel J, et al. "Type of mask may impact on continuous positive airway pressure adherence in apneic patients," PloS one, Vol 8, 64382, 2013

[27] Mortimore I, et al. "Comparison of nose and face mask CPAP therapy for sleep apnoea," Thorax, Vol. 53, 290-92, 1998

[28] Visscher $\mathrm{M}$, et al. "Face masks for noninvasive ventilation: fit, excess skin hydration, and pressure ulcers," Respiratory care, vol. 60, 1536-1547, 2015

[29] Worsley $P$, et al. "Investigating the effects of strap tension during non-invasive ventilation mask application: a combined biomechanical and biomarker approach" Medical devices, Vol 9, 409, 2016

** One of the few studies quantifying the facial pressures involved with strapping a CPAP mask

[30] Barros L, et al. "Facial pressure zones of an oronasal interface for noninvasive ventilation: a computer model analysis," Jornal Brasileiro de Pneumologia, vol. 40, .652657,2014

[31] Ebben M, Narizhnaya M, Segal A, Barone D, et al. "A randomised controlled trial on the effect of mask choice on residual respiratory events with continuous positive airway pressure treatment," Sleep medicine, Vol 15,619-624, 2014

[32] Ebben M, Oyegbile T, and Pollak C. "The efficacy of three different mask styles on a PAP titration night," Sleep medicine, Vol 13, 645-649, 2012

[33] Massie C and Hart R. "Clinical outcomes related to interface type in patients with obstructive sleep apnea/hypopnea syndrome who are using continuous positive airway pressure," CHEST Journal, Vol 123, 1112-18, 2003 
[34] Zhu X, Wimms A and Benjafield A. "Assessment of the performance of nasal pillows at high CPAP pressures" Journal of clinical sleep medicine, vol 9, 873, 2013

* Shows a systematic approach to verify the effect of nasal pillow CPAP interfaces

[35] De Araujo M, Vieira S, Vasquez E and Fleury B. "Heated humidification or face mask to prevent upper airway dryness during continuous positive airway pressure therapy," CHEST Journal, Vol 117, 142-147, 2000

[36] Balk EM, Moorthy D, Obadan NO, et al. "Diagnosis and treatment of obstructive sleep apnea in adults," Agency for Healthcare Research and Quality, 2011

[37] Ryan S, Garvey J, Swan V and Behan R. "Nasal pillows as an alternative interface in patients with obstructive sleep apnoea syndrome initiating continuous positive airway pressure therapy" Journal of sleep research, Vol 20, 367-73, 2011

[38] Newnam K, McGrath J, Estes T, et al. "An integrative review of skin breakdown in the preterm infant associated with nasal continuous positive airway pressure," Journal of Obstetric, Gynecologic, \& Neonatal Nursing, Vol 42, 508-516, 2013

[39] Beecroft J, Zanon S, Lukic D, et al. "Oral continuous positive airway pressure for sleep apnea: effectiveness, patient preference, and adherence," Chest, Vol 124, p.22002208, 2003

[40] Smith P, O'Donnell C, Allan L, et al. "A physiologic comparison of nasal and oral positive airway pressure," Chest, Vol 123, 689-694, 2003

[41] Anderson F, Kingshott R, Taylor D, et al. "A randomized crossover efficacy trial of oral CPAP (Oracle) compared with nasal CPAP in the management of obstructive sleep apnea," Sleep , Vol 26, 721-26, 2003

[42] Khanna R. , and Kline L. "A prospective 8 week trial of nasal interfaces vs. a novel oral interface (Oracle ${ }^{\mathrm{TM}}$ ) for treatment of obstructive sleep apnea hypopnea syndrome" Sleep medicine, Vol 4, 333-38, 2003

[43] Holanda M, Reis R, Winkeler G, et al. "Influence of total face, facial and nasal masks on short-term adverse effects during noninvasive ventilation" Jornal Brasileiro de Pneumologia, Vol 35, 164-173, 2009

[44] Brusasco C, Corradi F, De Ferrari A, et al. "CPAP Devices for Emergency Prehospital Use: A Bench Study" RESPIRATORY CARE, 04134, 2015

[45] Ma Z, Munguia J, Hyde, $P$ et al. "Design, Manufacture and Evaluation of Customized CPAP Breathing," in 29th SFFF Symposium, Austin Texas, US, 2018

[46] Orsted $\mathrm{H}$, Ohura $\mathrm{T}$ and Harding $\mathrm{K}$. "Pressure, shear, friction and microclimate in context," INTERNATIONAL REVIEW: PRESSURE ULCER PREVENTION. Wounds International, 2010

[47] Institute for Clinical and Economic Review. "Diagnosis and Treatment of Obstructive Sleep Apnea in Adults," The New England Comparative Effectiveness Public Advisory Council, 2013

[48] Trevino R. "Sleep Apnea \& the Eye" OD Rosenberg School of Optometry University of the Incarnate Word, 2011 
[49] Nasca T. "The Importance of CPAP Humidification" American Sleep Apnea Association, 2015

[50] Nava S, Navalesi P and Gregoretti C. "Interfaces and humidification for noninvasive mechanical ventilation," Respir Care , vol. 54, 71-84 , 2009

[51] Kohls D. "Clinical follow-up of Positive Airway Pressure (PAP) therapy," in Wisconsin Sleep Society, 2016

[52] Maruccia M, Ruggieri M and Onesti M. "Facial skin breakdown in patients with non-invasive ventilation devices: report of two cases and indications for treatment and prevention," International wound journal, Vol 12, 451-455, 2015

[53] NICE, "National Institute for Health and Care Excellence (NICE)," Available: https://www.nice.org.uk/about, 2018

[54] Association for Respiratory Technology and Physiology (ARTP). "Standards of Care - CPAP Devices (Technical and Performance Review). Version1, 2017

[55] Association for Respiratory Technology and Physiology (ARTP), "ARTP Sleep Standards of Care, Available: http://www.artp.org.uk/en/sleep/sleep-standards/ 2018.

[56] Morrison R, Van Koevering K, Nasser $\mathrm{H}$, et al. "Personalized 3D-Printed CPAP Masks Improve CPAP Effectiveness in Children with OSA and Craniofacial Anomalies" Combined Otolaryngology Spring Meetings, Vol 22: 26, 2015

** One of the few studies where custom CPAP mask development has been documented

57] Green G. "3D-Printed CPAP Masks for Children with Obstructive Sleep Apnea," National Library of Medicine, 2017

[58] Luna-Paredes C, Antón-Pacheco JL, García-Hernández G, et al. "Screening for symptoms of obstructive sleep apnea in children with severe craniofacial anomalies: assessment in a multidisciplinary unit," Int J Pediatr Otorhinolaryngol, vol. 76, 2012

[59] Plomp RG, Bredero-Boelhouwer HH, Joosten KF, et al. "Obstructive sleep apnoea in Treacher Collins syndrome: prevalence, severity and cause" Int $\mathrm{J}$ Oral Maxillofac Surg, vol 41, 696-701, 2012

[60] Rulon, S. Custom 3-D Printed Noninvasive Ventilation Mask for Children. Children's Hospital Medical Center, Cincinnati, US. Available: https://clinicaltrials.gov/ct2/show/NCT02896751, 2016

[61] Cheng $\mathrm{Y}$, and Chu J. "Application of rapid tooling to manufacture customized nasal mask cushion for continuous positive airway pressure (CPAP) devices." Rapid Prototyping Journal, Vol 19, 4-10, 2013

[62] Munguia $\mathrm{J}$ and $\mathrm{Ma} \mathrm{Z}$. "Design considerations to prevent pressure ulcers: optimising the respiratory mask interface," in TVS: Tissue Viability Society conference, Newcastle, UK, 2018

[63] Bitterman $\mathrm{BH}$. "Application of finite element modeling and analysis to the design of positive pressure oxygen masks" Air Force Institute of Technology, Wright-Patterson Air Force Base, Fairborn (OH), 1991 
[64] Yang JD, Dai J and Zhuang Z. "Simulating the interaction between a respirator and a head form using LS-DYNA.," Comp Aided Des Appl, vol 6, 539-51, 2009

[65] Piccione D and Moyer ET. "Modeling the Interface between a respirator and the human face" Adelphi (MD): Army Research Laboratory, 1997

[66] Wohlers T. "Additive Manufacturing State of the Industry. Annual Worldwide Progress Report”, Fort Collin: Wohlers Associates, 2008

[67] Ventola C. "Medical Applications for 3D Printing: Current and Projected Uses" Pharmacy and Therapeutics, vol. 39, 704-711, 2014

[68] Schubert C, Van Langeveld M and Donoso L. "Innovations in 3D printing: a 3D overview from optics to organs" Br J Ophthalmol, vol. 98, 159-161, 2014

[69] Banks J. "Adding value in additive manufacturing: Researchers in the United Kingdom and Europe look to 3D printing for customization" IEEE Pulse, vol 4, 22-26, 2013

[70] Wohlers T, Campbell I, Diegel O, et al. "Wohlers Report 2018" Wohlers Associates, Inc, 2018

[71] Conner B, Manogharan G, Martof A, et al. "Making sense of 3-D printing: Creating a map of additive manufacturing products and services," Additive Manufacturing, 1 , Vol 64-76, 2014

[72] 3DPrintingindustry, "METAMASON RAISED \$3M," METAMASON RAISED \$3M, 2016. Available: https://3dprintingindustry.com/news/metamason-raised-3m-81551/

[73] Inspirate Medical Pte Ltd Available: http://inspiratemedical.com

[74] FDA, "3D Printing of Medical Devices," U.S. Food and Drug Administration, 2018. Available:

https://www.fda.gov/MedicalDevices/ProductsandMedicalProcedures/3DPrintingofMedi calDevices

[75] PlasticsToday, "FDA tackles opportunities, challenges, of 3D printed medical devices," Plastics Today. Available: http://www.plasticstoday.com/articles/FDA-tacklesopportunities-challenges-3D-printed-medical-devices-140602

[76] Munguia $J$ et al. "3D PRINTING ENABLED-REDISTRIBUTED MANUFACTURING OF MEDICAL DEVICES," in Solid Freeform Fabrication 2016: Proceedings of the 26th Annual International. Solid Freeform Fabrication Symposium, Austin, 2016 


\section{TABLES AND FIGURES}

Table 1. Characteristics of different types of CPAP interfaces [15, 22, 23]

Table 2. Typical interface-related facial effects [28, 30, 35, 33, 38]

Table 3. Mask design requirements and considerations [54]

Figure 1. Airways opened during normal breathing

Figure 2. Obstructed breathing as a result of soft tissue collapsing

Figure 3 CPAP system schematic: 1) Headgear, 2) CPAP mask, 3) Connection, 4) Patient connection port, 5) Tubing, 6)Gas output port connector, 7)CPAP equipment (with or without humidifier) The exhaust port can be located in the connecting element (3) or the mask (2)

Figure 4 Working principle of CPAP therapy with pressurized air unblocking the collapsed soft tissue 